\title{
BMJ Open Barriers and facilitators of rendering HIV services by community health workers in sub-Saharan Africa: a meta- synthesis protocol
}

\author{
Gugulethu Eve Khumalo (D) ,1,2 Elizabeth Lutge, ${ }^{3}$ Praba Naidoo, ${ }^{4}$ \\ Tivani Phosa Mashamba-Thompson (D) ${ }^{5}$
}

To cite: Khumalo GE, Lutge E, Naidoo P, et al. Barriers and facilitators of rendering HIV services by community health workers in sub-Saharan Africa: a metasynthesis protocol. BMJ Open 2020;10:e037876. doi:10.1136/ bmjopen-2020-037876

- Prepublication history and additional material for this paper is available online. To view these files, please visit the journal online (http://dx.doi.org/10. 1136/bmjopen-2020-037876).

Received 20 February 2020 Revised 11 September 2020 Accepted 13 September 2020

Check for updates

(C) Author(s) (or their employer(s)) 2020. Re-use permitted under CC BY-NC. No commercial re-use. See rights and permissions. Published by BMJ.

For numbered affiliations see end of article.

Correspondence to Gugulethu Eve Khumalo; jcatcalvary@gmail.com

\section{ABSTRACT}

Introduction In sub-Saharan Africa (SSA), Human Immunodeficiency Virus (HIV) is the leading cause of morbidity and mortality. Community healthcare workers (CHWs) worldwide have been reported to be effective in strengthening the HIV programme by providing services such as adherence support, HIV education and safe sex education as part of their roles. The main aim of this metasynthesis is to synthesise qualitative evidence on studies that have been conducted in SSA countries to understand the barriers to and facilitators of providing HIV services by CHWs across all settings

Methods and analysis This meta-synthesis will be guided by Preferred Reporting Items for Systematic Reviews and Meta-Analyses. An initial search was conducted on 15 November 2019 for studies published between 2009 and 2019 using the population exposure outcome nomenclature. We searched the EBSCOHost(ERIC; Health Source-Nursing/Academic Edition), Google Scholar and PubMed databases for the relevant studies. The Ritchie and Spencer framework will be used for data synthesis and the Supporting the Use of Research Evidence Framework analysis will be used to analyse data. We will conduct critical appraisal on selected studies using the Qualitative Assessment and Review Instrument to limit risk of bias.

Ethics and dissemination This review does not involve any human participants and therefore ethical approval will not be required. We will publish the protocol as well as the findings in any relevant journal and various media namely conferences; symposia, local and international health stakeholders.

PROSPERO registration number CRD42020160012. Conclusion Evidence from this review will provide synthesised evidence to the utilisation of CHWs in HIV services in SSA.

\section{INTRODUCTION}

As reported, sub-Saharan African (SSA) countries have the highest accumulated percentage of HIV infections globally. ${ }^{12}$ The HIV programmes in SSA are programmes that focus on preventing the transmission of HIV through a combination of behavioural, biomedical and structural strategies. ${ }^{3}$
Strengths and limitations of this study

- The strength of this meta-synthesis lies in that it is the first to review the barriers to and facilitators of providing HIV services by the community health workers (CHWs) in sub-Saharan Africa in the past 10 years.

- In sub-Saharan Africa, HIV prevalence continues to rise due to people with the virus living longer through effective antiretroviral therapy (ART). Therefore, task shifting to reduce the workload burden on the other health workers should be considered by effectively using CHWs to promote ART adherence, thereby avoiding opportunistic infections as result of disease progression.

- The study is limited only to published qualitative research studies and not, for example grey literature, unpublished reports and government documents.

- The systematic review also excludes any qualitative, published studies that are in any language other than English because of limited financial resources to cover interpretation costs.

- Both these limitations will be mitigated by working with an experienced librarian to ensure that the meta-synthesis includes as many suitable, highquality studies as possible.

The effectiveness of community health workers (CHWs) in strengthening the HIV programme is evident in $\mathrm{SSA}^{45}$ which is home to $12 \%$ of the global population, yet accounts for $71 \%$ of the global burden of HIV infection. ${ }^{2}$ However, the gap between the need for antiretroviral therapy (ART) and actual provision of ART is still wide in SSA. ${ }^{6}$ This gap is due to shortfalls in HIV testing, access to ART and long-term retention in care of which the CHWs can close by encouraging HIV testing, referring communities that need ART to healthcare and education on adherence. ${ }^{6}$ It is then important to answer the research question of this review to better understand the barriers to and facilitators of providing HIV 
services by the CHWs so that appropriate interventions can be implemented in different settings of this region. There have been studies including systematic reviews that have described the HIV services provided by CHWs in SSA. However, after extensive search, no meta-synthesis was found which reported on the barriers to and facilitators of providing HIV services by the CHWs in SSA in the past 10 years, that is, from 2009 to 2019.

In the past 8-10 years, several SSA countries became more serious about using CHWs as part of the task-shifting concept to address the shortage of the health workers and the growing burden of the HIV epidemic among the communities. ${ }^{7-17}$ Moreover, in 2011, the World Health Organization (WHO) provided the first cost guidance for developing a locally adaptable and nationally scalable CHW system within primary healthcare systems in SSA. ${ }^{18}$ This meta-synthesis will share light on what has worked and what has not worked in terms of using this cadre in providing HIV services including ART.

The antiretroviral treatment (ART) has improved the lives of people in SSA but yet a substantial percentage, sometimes as high as $60 \%$ of people living with HIV (PLWH) are not on ART. ${ }^{1}$ CHWs are an integral part of both the community and the health system and hence are at an advantage of being the first line of contact with PLWH and being their link to the health system for continuum of care.

Before 2000, CHWs were not an integral part of the health system in most SSA countries ${ }^{19}$ but since 2000 , they shifted from providing voluntary health services to communities, to being an integral part of the health system ${ }^{519}$ although not fully integrated. ${ }^{6}$ The HIV services provided by CHWs vary in each of the SSA countries. These included patient support (counselling, home-based care, education, adherence support and livelihood support) and health service support (screening, referral and health service organisation and surveillance) ${ }^{520} \mathrm{Commu}-$ nity health workers also enhance the reach, uptake and quality of HIV services, as well as the dignity, quality of life and retention in care of PLWH. ${ }^{5}$ They certainly are a potential solution in overcoming challenges faced by the HIV programme in the sub-Saharan countries. ${ }^{51}$ However, CHWs are still not effectively used to play this role because they are not fully integrated into the health system. ${ }^{6}$ There is still no harmonised approach in their employment, remuneration, training, management, supervision and retention which pose barriers for their utilisation to provide HIV services in SSA. ${ }^{62-24}$

In most SSA countries, CHWs are employed without job descriptions which causes ambiguity around their roles and ultimately leads to them conducting tasks outside their training. ${ }^{23}$ In terms of having standardised protocols and defined roles for this cadre, countries like Bangladesh and Brazil that SSA can be used as a benchmark. ${ }^{625}$

Political structures in SSA have an opportunity to strengthen the HIV programme and decrease HIV infections by effectively utilising CHWs. However, there is insufficient evidence on the barriers to and facilitators of
CHWs' role in HIV care, resulting in confirmed ineffective utilisation of this cadre.

\section{STUDY AIM}

The aim of this meta-synthesis is to synthesise qualitative evidence which explores the barriers to and facilitators of rendering HIV specific services by the CHWs.

\section{Objectives}

- To synthesise evidence on the barriers to providing HIV services by CHWs in SSA.

- To synthesise evidence on the facilitators of providing HIV services by CHWs in SSA.

\section{METHODS AND ANALYSIS}

To synthesise evidence on published qualitative studies regarding the barriers to and facilitators of rendering HIV services by the CHWs, we will conduct a metasynthesis, systematic review. We will be guided by the Preferred Reporting Items for Systematic Reviews and Meta-Analyses (PRISMA). ${ }^{26-28}$ The meta-synthesis will be guided by an interpretive paradigm which aims for researchers to distance themselves from their own views and ideas. ${ }^{29-31}$ We selected this paradigm because it guides the researchers to use the experience of participants or of reported data to construct and interpret their understanding from collected data without tainting it with their own views or experiences. ${ }^{29-31}$ We will use all included qualitative studies regardless of the methodology used in the study (eg, phenomenology, content analysis, anthropology etcetera). We will use the Ritchie and Spencer framework analysis ${ }^{32}$ and the Supporting the Use of Research Evidence (SURE) Framework, ${ }^{33}$ which will be our framework for thematic synthesis. We will use NVivo V.12 Pro to code themes emerging from the extracted data. The report of findings for this review will be published as per PRISMA guidelines. ${ }^{27}$

The research question of our systematic review and meta-synthesis is: What are the barriers to and facilitators of rendering HIV services by CHWs in SSA?

We will use the population exposure outcome mnemonic $^{34}$ to determine suitability of our research question to be answered using a meta-synthesis (table 1).

Box 1 indicates the search strategy example from PubMed, including planned limits.

\section{Eligibility criteria}

Inclusion criteria

We will include:

- Studies that are in the English language because of limited financial resources for translation.

- Studies that are qualitative or mixed method as long as there is a qualitative component regardless of the study design.

- Studies published in the past 10 years (between 2009 and 2019). 
Table 1 PEO showing suitability of our research question for a chosen method

\begin{tabular}{llll} 
& Population (P) & Exposure (E) & Outcome (O) \\
\hline Keywords & Community health worker & Rendering HIV services in communities & Barriers of rendering or offering HIV \\
& Lay health worker & & Services \\
& Village doctor & & Facilitators of rendering or offering HIV \\
Community care worker & Sommunity caregiver & & \\
& Home-based carer & & \\
& Lay health workers & & \\
& Lay counsellors & & \\
& Community volunteers &
\end{tabular}

- Studies with evidence where CHWs were assigned to offer only HIV health services regardless of their work titles or if they were assigned to offer multiple health services as long as these health services also included HIV services.

- Studies that have evidence of barriers to and facilitators of rendering HIV services by the CHWs.

- Studies conducted only in SSA countries.

\section{Exclusion criteria}

- All quantitative studies that are related to HIV services offered by the CHWs.

- Qualitative studies regarding the topic published before 2009 or after 2019.

- All qualitative documents that are in grey literature.

- Other healthcare workers that are assigned to provide HIV services in the community.

- Other health services that are provided by CHWs that are non-HIV related.

\section{Search strategy: information sources}

On the 15 November 2019, we searched the following databases: EBSCOHost- (ERIC; Health Source-Nursing/ Academic Edition; Medline) and Google Scholar. We conducted a Boolean search and also Medical Subject Headings search using keywords to search for relevant studies to answer our research question. The keywords we used were: CHWs; lay health workers; HIV services;

\section{Box 1 Search strategy example (PubMed)}

(("hiv"(MeSH Terms) OR "hiv"(All Fields)) AND services(All Fields)) AND ("community health workers"(MeSH Terms) OR ("community"(All Fields) AND "health"(All Fields) AND "workers"(All Fields)) OR "community health workers"(All Fields)) OR (lay(All Fields) AND ("health"(MeSH Terms) OR "health"(All Fields)) AND workers(All Fields)) AND ("qualitative research"(MeSH Terms) OR ("qualitative"(All Fields) AND "research"(All Fields)) OR "qualitative research"(All Fields) OR ("qualitative"(All Fields) AND "study"(All Fields)) OR "qualitative study"(All Fields)) AND ("africa south of the sahara"(MeSH Terms) OR ("africa"(All Fields) AND "south"(All Fields) AND "sahara"(All Fields)) OR "africa south of the sahara"(All Fields) OR ("sub"(All Fields) AND "saharan"(All Fields) AND "africa"(All Fields)) OR "sub saharan africa"(All Fields)) AND ((“2009/01/01"(PDAT) : “2019/11/31"(PDAT)) AND “humans"(MeSH Terms) AND English(lang)). barriers; facilitators and SSA as outlined in table 1. The search results were entered on a Search Form with search dates, database names and the results of the search (online supplemental appendix 1). Studies that will come from the reference list of one of the studies selected will also be searched.

\section{Screening of articles}

The articles for this systematic review will be screened by two independent reviewers to increase trustworthiness and to eliminate bias. The reviewers will use the inclusion and exclusion criteria to select relevant studies. The screening of studies will be done in three stages using EndNote $\mathrm{X} 9$ as a reference manager. If there is any disagreement between the reviewers especially during the last stage of screening (for full-text studies), a third reviewer will be used to help resolve the disagreement. The first stage was initiated by running a search on the relevant databases to screen for titles (online supplemental appendix 1) and all the relevant titles were saved on the reference manager. For the second stage the reviewers will screen the abstracts of the selected titles using the Abstract Selection Form (online supplemental appendix 2). The reviewers will select abstracts and thereafter meet to discuss selected abstracts and resolve any disagreements. The final selected abstracts will be taken to the third stage which involves full-text screening of the studies using fulltext screening form (online supplemental appendix 3). The reviewers will obtain full articles from the relevant sources including from authors if not publically available. Final studies included after the third stage will be the final number of studies that will be included for the metasynthesis. We do not anticipate bias because of missing information on the selected studies as all the selected studies would have met our inclusion criteria. However, if there is any missing information on the selected final full-text articles, the authors will be contacted to clarify and given a time frame for feedback which will be mostly a month. If the authors do not respond within a month, then the studies will be excluded from this review to eliminate bias. A $\log$ will be written for studies that were excluded from the meta-synthesis and the reasons for their exclusion will be explained. The final included 
qualitative studies will each be given a unique ID number. The PRISMA 2009 flow diagram ${ }^{26}$ will be used to report the screening results.

\section{Risk of bias: assessing the quality of selected studies}

Critical appraisal in meta-synthesis is for assessing the rigour on the selected qualitative studies and to also ensure that they are free from methodological issues that may somehow affect the findings of the meta-synthesis. ${ }^{35} 36$ We will use the Qualitative Assessment and Review Instrument (QARI) developed by the Joanna Briggs Institute, Australia $^{37}$ (online supplemental appendix 4) to appraise the quality of the selected studies. This will be undertaken by two independent reviewers. We will develop a scoring system for rating the quality of studies. The scoring system and the QARI tool will be piloted on ten percent of studies that were selected for the meta-synthesis. This will assist us in examining the scores provided; to inform the development of an appropriate scoring system and to eventually determine the cut-off points for low and highquality studies. Where there is disagreement between scoring results, a third independent reviewer will be used for arbitration.

\section{Data extraction}

We will develop a data extraction tool using Google Forms for all studies selected for the meta-synthesis. Once the form has been created, the form will be pretested or piloted by at least two independent individuals on five included full-text studies. The data extraction form will contain the following information about the selected studies: author and year; title; aim; study design; study setting; study population; percentage of target population; exposure (rendering of HIV services) and outcome (barriers to or facilitators of providing HIV services) (online supplemental appendix 5). A short, structured questionnaire will be emailed to the authors to complete any missing data or information. The authors will be followed up at least for 1 month until they provide the missing information. The study will be excluded if the authors do not send the missing information.

\section{Data synthesis}

The aim of data synthesis or analysis is to gather all the findings from all the selected studies into a meaningful statement or a set of statements which will represent and explain the phenomenon under study or answer the research question of the systematic review. ${ }^{38-40}$ The synthesis or analysis will be conducted by two research team members independently to guard against bias and improve trustworthiness. The two members are both experts in thematic and content analysis using NVivo V.12 Pro. The SURE Framework analysis ${ }^{33}$ (online supplemental appendix 6) will be used to analyse data and for improving comparability and trustworthiness of the study. The SURE framework can be applied to any healthcare research ${ }^{41}$ and the research team agreed on this framework analysis because of its domains that are linked to factors affecting health service delivery. ${ }^{41}$ The data synthesis approach that we will use is the same approach that was used in other studies. ${ }^{42}{ }^{43}$ We will juxtapose sources of evidence, that is, when data about the barriers to and facilitators of providing HIV services are extensively mentioned in one paper, that information will allow insight into those barriers and facilitators in another paper.

The Ritchie and Spencer framework analysis which consist of five stages of thematic analysis will guide the analysis of the study. ${ }^{32}$ This framework analysis method will be selected because it is appropriate for thematic analysis of textual data, where it is important to be able to compare and contrast data by themes across many studies while also situating each perspective in context by retaining the connection to other aspects of each study's account. ${ }^{44}$ The five stages of analysis are familiarisation, generating themes, searching for themes, reviewing themes and finally producing a report. ${ }^{32}$ The first stage is familiarisation, where the data analyst will familiarise himself with the selected studies and immerses himself in them while writing down recurrent themes and ideas. The second stage is identifying a thematic framework where the evolving themes are written down and form part of the study thematic framework that is temporal as it can change at different parts of data analysis. The thematic framework then guides the allocation of the study contents to written themes. We decided to use the SURE framework for this stage. The third stage which is indexing involves identifying portions from the studies and allocating theme numbers to them. This will be done using NVivo V.12 Pro. The fourth stage which is charting entails extracting the portions that were allocated theme numbers and placing them under themes and subthemes that were created during thematic framework identification. The fifth and the last stage is mapping and interpretation which is when the key characteristics of the charts are analysed to guide the interpretation of the data sets and to ultimately produce a report of findings. The PRISMA standards will also guide the writing of the report.

\section{Patient and public involvement}

Patients and the public were not involved in the design and planning of this study.

\section{Publishing the protocol}

We will disseminate the findings of this meta-synthesis through publication in a peer-reviewed journal; relevant conference; local and international health government departments. Publication of this meta-synthesis will enable transparency of the review methodology and allows feedback and views of the peer review before the review commences. This will also improve quality, trustworthiness of this review. Publication will also prevent duplication in case other researchers want to conduct a similar review. 


\section{DISCUSSION}

Globally, SSA region is the most affected by the HIV epidemic and ${ }^{145}$ with a large percentage still not having access to proper healthcare and treatment. In this same region, CHWs are increasingly used to provide HIV services and there is evidence that they play a role in improving HIV programmes through various community interventions. ${ }^{46-48}$ However, there is high variability of CHWs' utilisation between different sub-Saharan countries. ${ }^{47}$ Therefore, synthesising evidence of what has worked and what has not worked in the provision of HIV services by CHWs is important for benchmarking and adapting or adopting by similar country settings. The results of this systematic review should then have practical implications for future clinical practice, research and policy. The past 10 years, from 2009 to 2019, CHWs have been increasingly mobilised to improve health systems with the WHO issuing guidelines on affordable strategies to implement their programme in $2011 .^{18} 49$ This meta-synthesis, therefore, excludes qualitative studies that were published before 2009 and after 2019. The other limitation of this meta-synthesis is the exclusion of other reports and documents that may have had evidence on the topic but were published in grey literature and also not published as qualitative studies in the selected databases and journals. However, working with the librarian ensured that as many databases and journals relevant to the topic were searched to include as many studies as possible.

\section{Author affiliations}

${ }^{1}$ Discipline of Public Health Medicine, University of KwaZulu-Natal College of Health Sciences, Durban, South Africa

${ }^{2}$ Department of Health, KwaZulu-Natal, Pietermaritzburg, KwaZulu-Natal, South Africa

${ }^{3}$ KwaZulu Natal Department of Health, South African Government, Durban, KwaZulu Natal, South Africa

${ }^{4}$ Library, University of KwaZulu-Natal - Medical School, Durban, South Africa

${ }^{5}$ Public Health, University of KwaZulu Natal, Durban, KwaZulu Natal, South Africa

Acknowledgements The authors express their gratitude to the College of Health Science, University of KwaZulu-Natal for support of this study.

Contributors The protocol was conceptualised and developed by GEK and TPM-T. The subsequent study, review of abstracts, full article studies and extraction process will comprise of all team members (GEK, TPM-T, EL and PN).

Funding The authors have not declared a specific grant for this research from any funding agency in the public, commercial or not-for-profit sectors.

Competing interests None declared.

Patient consent for publication Not required.

Provenance and peer review Not commissioned; externally peer reviewed.

Supplemental material This content has been supplied by the author(s). It has not been vetted by BMJ Publishing Group Limited (BMJ) and may not have been peer-reviewed. Any opinions or recommendations discussed are solely those of the author(s) and are not endorsed by BMJ. BMJ disclaims all liability and responsibility arising from any reliance placed on the content. Where the content includes any translated material, BMJ does not warrant the accuracy and reliability of the translations (including but not limited to local regulations, clinical guidelines, terminology, drug names and drug dosages), and is not responsible for any error and/or omissions arising from translation and adaptation or otherwise.

Open access This is an open access article distributed in accordance with the Creative Commons Attribution Non Commercial (CC BY-NC 4.0) license, which permits others to distribute, remix, adapt, build upon this work non-commercially, and license their derivative works on different terms, provided the original work is properly cited, appropriate credit is given, any changes made indicated, and the use is non-commercial. See: http://creativecommons.org/licenses/by-nc/4.0/.

ORCID iDs

Gugulethu Eve Khumalo http://orcid.org/0000-0002-1881-4104

Tivani Phosa Mashamba-Thompson http://orcid.org/0000-0002-4193-2416

\section{REFERENCES}

1 Dwyer-Lindgren L, Cork MA, Sligar A, et al. Mapping HIV prevalence in sub-Saharan Africa between 2000 and 2017. Nature 2019;570:189-93.

2 Kharsany ABM, Karim QA. Hiv infection and AIDS in sub-Saharan Africa: current status, challenges and opportunities. Open AIDS J 2016;10:34-48.

3 Organization WH. Essential prevention and care interventions for adults and adolescents living with HIV in resource-limited settings, 2008.

4 Ngilangwa DP, Mgomella GS. Factors associated with retention of community health workers in maternal, newborn and child health programme in Simiyu region, Tanzania. Afr J Prim Health Care Fam Med 2018;10:1-8.

5 Mwai GW, Mburu G, Torpey K, et al. Role and outcomes of community health workers in HIV care in sub-Saharan Africa: a systematic review. J Int AIDS Soc 2013;16:18586-86.

6 Hermann K, Van Damme W, Pariyo GW, et al. Community health workers for ART in sub-Saharan Africa: learning from experience-capitalizing on new opportunities. Hum Resour Health 2009;7:31.

7 Banteyerga $\mathrm{H}$. Ethiopia's health extension program: improving health through community involvement. MEDICC Rev 2011;13:46-9.

8 Chang LW, Kagaayi J, Nakigozi G, et al. Effect of peer health workers on AIDS care in Rakai, Uganda: a cluster-randomized trial. PLoS One 2010;5:e10923.

9 Islam MM, Conigrave KM. HIV and sexual risk behaviors among recognized high-risk groups in Bangladesh: need for a comprehensive prevention program. Int J Infect Dis 2008;12:363-70.

$10 \mathrm{Kim} \mathrm{MH}$, Ahmed S, Buck WC, et al. The Tingathe programme: a pilot intervention using community health workers to create a continuum of care in the prevention of mother to child transmission of HIV (PMTCT) cascade of services in Malawi. J Int AIDS Soc 2012;15 Suppl 2:17389.

11 Ledikwe JH, Kejelepula M, Maupo K, et al. Evaluation of a wellestablished task-shifting initiative: the lay counselor cadre in Botswana. PLoS One 2013;8:e61601.

12 Maes K, Kalofonos I. Becoming and remaining community health workers: perspectives from Ethiopia and Mozambique. Soc Sci Med 2013;87:52-9.

13 Rich ML, Miller AC, Niyigena P, et al. Excellent clinical outcomes and high retention in care among adults in a community-based HIV treatment program in rural Rwanda. J Acquir Immune Defic Syndr 2012;59:e35-42.

14 Department of Health South Africa. Policy Framework and Strategy for Ward Based Primary Healthcare Outreach Teams (2018/92023/2024). In: Department of health South Africa, 2018.

15 Johnson CD, Noyes J, Haines A, et al. Learning from the Brazilian community health worker model in North Wales. Global Health 2013;9:25.

16 Rachlis B, Naanyu V, Wachira J, et al. Community perceptions of community health workers (CHWs) and their roles in management for HIV, tuberculosis and hypertension in Western Kenya. PLoS One 2016;11:e0149412.

17 Rahman R, Pinto RM, Zanchetta MS, et al. Delivery of communitybased care through Inter-professional teams in Brazil's unified health system (UHS): comparing perceptions across community health agents (CHAs), nurses and physicians. J Community Health 2017;42:1187-96.

18 McCord GC, Liu A, Singh P. Deployment of community health workers across rural sub-Saharan Africa: financial considerations and operational assumptions. Bull World Health Organ 2013;91:244-53.

19 Singh P, Sachs JD. 1 million community health workers in subSaharan Africa by 2015. Lancet 2013;382:363-5.

20 Naidoo N, Matlakala N, Railton J, et al. Provision of HIV services by community health workers should be strengthened to achieve full programme potential: a cross-sectional analysis in rural South Africa. Trop Med Int Health 2019;24:401-8.

21 Mundeva $\mathrm{H}$, Snyder J, Ngilangwa DP, et al. Ethics of task shifting in the health workforce: exploring the role of community health workers 
in HIV service delivery in low- and middle-income countries. BMC Med Ethics 2018;19:71.

22 Kok MC, Vallières F, Tulloch $\mathrm{O}$, et al. Does supportive supervision enhance community health worker motivation? A mixed-methods study in four African countries. Health Policy Plan 2018;33:988-98.

23 Schuster RC, McMahon DE, Young SL. A comprehensive review of the barriers and promoters health workers experience in delivering prevention of vertical transmission of HIV services in sub-Saharan Africa. AIDS Care 2016;28:778-94.

24 Standing $\mathrm{H}$, Chowdhury AMR. Producing effective knowledge agents in a pluralistic environment: what future for community health workers? Soc Sci Med 2008;66:2096-107.

25 Perry HB, Zulliger R, Rogers MM. Community health workers in low-, middle-, and high-income countries: an overview of their history, recent evolution, and current effectiveness. Annu Rev Public Health 2014;35:399-421.

26 Liberati A, Altman DG, Tetzlaff J, et al. The PRISMA statement for reporting systematic reviews and meta-analyses of studies that evaluate health care interventions: explanation and elaboration. PLoS Med 2009;6:e1000100.

27 Moher D, Altman DG, Liberati A, et al. PRISMA statement. Epidemiology 2011;22:128.

28 Moher D, Liberati A, Tetzlaff J, et al. Preferred reporting items for systematic reviews and meta-analyses: the PRISMA statement. Ann Intern Med 2009;151:264-9.

29 Blaikie N, Priest J. Social research: paradigms in action. Cambridge, UK: Polity Press, 2017.

30 Günbayi I, Sorm S. Social paradigms in guiding social research design: the functional, interpretive, radical humanist and radical structural paradigms. International Journal on New Trends in Education and Their Implications 2018;9.

$31 \mathrm{R}$. Ponelis S. Using interpretive qualitative case studies for exploratory research in doctoral studies: a case of information systems research in small and medium enterprises. International Journal of Doctoral Studies 2015;10:535-50.

32 Ritchie J, Spencer L. Qualitative data analysis for applied policy research. In: The qualitative researcher's companion. Sage, 2002: 305-29.

33 The SURE Collaboration. SURE Guides for preparing and using evidence-based policy Brief 5: 5 Identifying and addressing barriers to implementing policy options, Version 2.1, 2011. Available: https:// www.who.int/evidence/sure/guides/en/ [Accessed 10 May 2019].

34 Butler A, Hall H, Copnell B. A guide to writing a qualitative systematic review protocol to enhance evidence-based practice in nursing and health care. Worldviews Evid Based Nurs 2016;13:241-9.
35 Barnett-Page E, Thomas J. Methods for the synthesis of qualitative research: a critical review. BMC Med Res Methodol 2009;9:59.

36 Finlayson KW, Dixon A. Qualitative meta-synthesis: a guide for the novice. Nurse Res 2008;15:59-71.

37 Pearson A. Balancing the evidence:incorporating the synthesis of qualitative data into systematic reviews. Int $J$ Evid Based Healthc 2004;2:45-64.

38 Jones T, Evans D. Conducting a systematic review. Aust Crit Care 2000;13:66-71.

39 Lucas PJ, Baird J, Arai L, et al. Worked examples of alternative methods for the synthesis of qualitative and quantitative research in systematic reviews. BMC Med Res Methodol 2007;7:4.

40 Munn Z, Tufanaru C, Aromataris E. JBI's systematic reviews: data extraction and synthesis. Am J Nurs 2014;114:49-54.

41 Wakida EK, Okello ES, Rukundo GZ, et al. Health system constraints in integrating mental health services into primary healthcare in rural Uganda: perspectives of primary care providers. Int J Ment Health Syst 2019;13:16.

42 Babovič M, Fu R-H, Monrouxe LV. Understanding how to enhance efficacy and effectiveness of feedback via e-portfolio: a realist synthesis protocol. BMJ Open 2019;9:e029173.

43 Brennan N, Bryce M, Pearson M, et al. Towards an understanding of how appraisal of doctors produces its effects: a realist review. Med Educ 2017;51:1002-13.

44 Gale NK, Heath G, Cameron E, et al. Using the framework method for the analysis of qualitative data in multi-disciplinary health research. BMC Med Res Methodol 2013;13:117-17.

45 Ramjee G, Daniels B. Women and HIV in sub-Saharan Africa. AIDS Res Ther 2013:10:30.

46 Smith S, Deveridge A, Berman J, et al. Task-shifting and prioritization: a situational analysis examining the role and experiences of community health workers in Malawi. Hum Resour Health 2014;12:24.

47 Taylor C, Griffiths F, Lilford R. Affordability of comprehensive community health worker programmes in rural sub-Saharan Africa. BMJ Glob Health 2017;2:e000391.

48 World Health Organization. Community health workers at the millennium villages project increase access to the health workforce, 2011. Available: https://www.who.int/workforcealliance/forum/2011/ hrhawardscs11/en/ [Accessed 12 Apr 2019].

49 Assegaai T, Schneider H. National guidance and district-level practices in the supervision of community health workers in South Africa: a qualitative study. Hum Resour Health 2019;17:25. 